\title{
Modeling the amorphous-to-crystalline phase transformation in network materials
}

\author{
K. Kohary,* V. M. Burlakov, and D. G. Pettifor \\ Department of Materials, University of Oxford, Parks Road, Oxford, OX1 3PH, United Kingdom
}

(Received 4 January 2005; published 9 June 2005)

\begin{abstract}
We have developed a computationally efficient rate equation model to study transformations between amorphous and crystalline phases of network forming materials. Amorphous and crystalline phases are treated in terms of their atomic ring distributions. The transformation between the two phases is considered to be driven by the conversion of one set of rings into another, following the Wooten-Winer-Weaire bond-switching algorithm. Our rate equation model describes both the generation and collapse of amorphous regions in thin crystalline films, the processes crucial for phase-change data storage materials. It is found that the amorphous spot collapse is assisted by the motion of certain crystal facets.
\end{abstract}

DOI: 10.1103/PhysRevB.71.235309

PACS number(s): 61.43.-j, 64.70.Kb

\section{INTRODUCTION}

Data storage devices utilizing the properties of phasechange materials are expected to play an important role in multimedia applications in the near future. ${ }^{1-5}$ Well-known phase-change materials are based on chalcogenide alloy systems, such as In-Sb-Te, Ge-Sb-Te, and Ag-In-Sb-Te. ${ }^{6} \mathrm{~A}$ bit of information can be stored in such materials by amorphizing/ crystallizing a small region by a short heat pulse due to a laser or electron beam or current. One of the key problems in phase-change material applications is the density of data storage, which is determined mainly by the bit size. To compete with other technologies the bit size in the new generation of phase-change data storage materials must be below $30 \mathrm{~nm}$. This implies 4 Gbyte capacity for a data storage module approximately the size of a coin.

Evaluating the amorphous/crystalline bit lifetime requires a reliable model describing the amorphous-to-crystalline phase transition. Traditional approaches involve either atomistic simulations using molecular dynamics ${ }^{7-12}$ and Monte Carlo methods, ${ }^{13,14}$ or continuum descriptions of the phase transformation. ${ }^{15-21}$ Atomistic simulations are computationally very expensive, so that they cannot be performed for large systems on a realistic time scale. Typically systems of only a few thousands atoms (system size $\sim 3-5 \mathrm{~nm}$ ) can be studied on the nanosecond scale. The continuum approach allows a much longer time scale to be accessed, but it has limited applicability on the nanometer scale, i.e., the scale of immediate interest to phase-change data storage applications. In order to address the problem on realistic time and length scales we have developed a model for treating the amorphous-to-crystalline phase transformation in network materials as a transformation between the distributions of atomic rings characteristic for each phase. The model combines atomistic and continuum approaches by using microscopic parameters for the ring distributions and energetics, but continuous ring concentration variables to describe the amorphous and crystalline phases.

\section{THE RATE EQUATION MODEL}

For illustrating our approach let us first consider a twodimensional (2D) network of atoms and bonds, in which all the atoms are four-fold coordinated (see Fig. 1). The crystalline phase in such a system is represented by a square lattice. We define an atomic ring as the shortest closed path through nearest-neighbor atoms with each bond passed only once. ${ }^{22}$ The crystalline structure is therefore characterized by a ring distribution, which contains only four-members rings, whereas an amorphous structure involves three-, four-, five-, etc., $k$-member rings. In any amorphous system the smallest ring $\left(N_{\min }\right)$ is a three-member one, but there is no particular reason to define a maximum ring size $\left(N_{\max }\right)$. The latter must be identified in each particular case. For instance, the largest ring size in simulated bulk amorphous silicon and amorphous carbon networks is 9 and 12, respectively. ${ }^{23,24}$

The ring distribution is a characteristic of the material topology. If the atomic coordination numbers are the same in both the amorphous and crystalline phases of a 2D network, then the total number of rings is conserved. The structural difference between the phases may, therefore, be attributed to their different ring distributions. Our model evaluates these distributions by assuming that the total energy of the system can be approximated by a sum over the elastic energy of all the different rings, namely

$$
E=\sum_{k=N_{\min }}^{N_{\max }} E_{k} n_{k},
$$

where $E_{k}$ and $n_{k}$ are the average elastic energy and ring concentrations of a $k$-member ring, respectively. All ring energies in our model are measured with respect to the fourmember ring. The ring energies may, therefore, be regarded as energy penalties with respect to the ground state crystalline phase.

To model the crystalline-to-amorphous transformation in a network material, we use the Wooten-Winer-Weaire (WWW) bond switching algorithm, ${ }^{25}$ which converts one set of atomic rings into another. According to this algorithm four atoms are chosen randomly to form a nearest-neighbor chain in the network, such as the atoms ABCD shown in Fig. 1. The WWW move involves breaking the two bonds $\mathrm{AB}$ and $\mathrm{CD}$, and creating two new bonds $\mathrm{AC}$ and $\mathrm{BD}$. Note that a WWW move conserves the original atomic coordination, as the atoms $\mathrm{ABCD}$ are chosen such that no double bonds or 

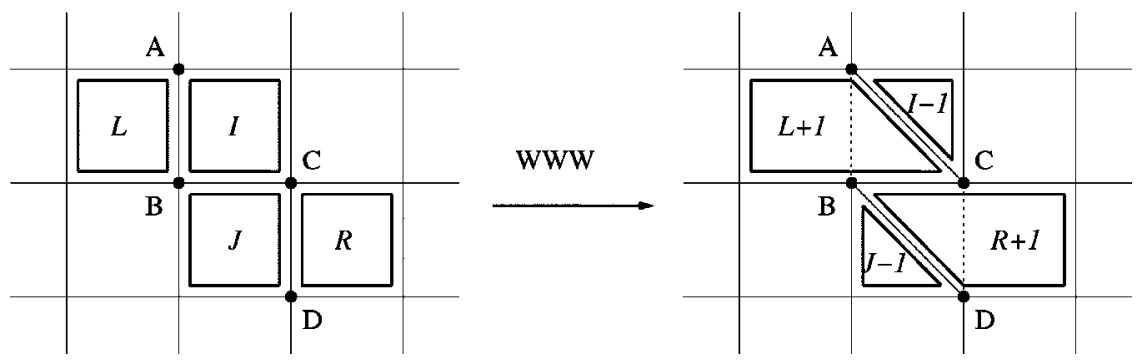

FIG. 1. A configurational change in the WWW method involving two neighbors A and D about the bond BC. Left-hand panel: schematic four four-member rings in crystalline phase. Right-hand panel: WWW bond switching event in which bonds AB and CD are broken and AC and BD created, thereby forming two three- and two five-member rings and changing the ring statistics. In general, the WWW move causes the size of a ring having the $\mathrm{BC}$ bond and one of the $\mathrm{AB}$ or $\mathrm{CD}$ bonds (rings $I$ and $J$ ) to decrease by one, but the size of the rings having only $\mathrm{AB}$ or $\mathrm{CD}$ bonds (rings $L$ and $R$ ) to increase by one.

bond crossing is generated after the bond switching. The new structure in our model is accepted with the probability defined by the relative rate,

$$
p_{\text {in }, \mathrm{fi}}=\exp \left(-\frac{E_{\mathrm{fi}}-E_{\text {in }}}{2 k_{B} T}\right)
$$

where $E_{\mathrm{fi}}$ and $E_{\mathrm{in}}$ are the energies of the final and initial states given by Eq. (1), $T$ is the system temperature, and $k_{B}$ is the Boltzmann constant. It follows from Eq. (2) that a high amorphous-to-crystalline transition enthalpy slows down the direct transformation but speeds up the reverse transformation. ${ }^{26}$ The symmetric form of the detailed balance given by Eq. (2) has been used in kinetic Monte Carlo simulations, ${ }^{27}$ as well as in analytical calculations. ${ }^{28,29}$

The difference between the initial and final energies in Eq. (2) can be expressed in terms of the energy penalties for the rings as follows. As we have seen from Fig. 1 the WWW move results in the size of the rings $I$ and $J$ decreasing by one, but the size of the rings $L$ and $R$ increasing by one. The corresponding change in energy is, therefore,

$$
E_{\mathrm{fi}}-E_{\mathrm{in}}=E_{L+1}+E_{R+1}+E_{I-1}+E_{J-1}-E_{L}-E_{R}-E_{I}-E_{J} .
$$

To derive the rate equation for the concentration of $k$-member rings, we introduce the probability $P_{\{L, I, J\}}$ of finding the ring configuration $\{L, I, J, R\}$. This probability depends on the concentrations of rings $n_{k}(t)(k=L, I, J$, and $R)$ at time $t$ and on the ring sizes, namely,

$$
P_{\{L, I, J, R\}}=\frac{1}{Z} \frac{2 L I J R}{(I-1)(J-1)} n_{L}(t) n_{I}(t) n_{J}(t) n_{R}(t),
$$

where $Z$ is a normalization factor. The configurational prefactor in Eq. (4) describes all possible permutations for a given $\{L, I, J, R\}$ configuration. The factor 2 reflects the fact that only two possibilities exist in two dimensions to arrange the atoms $\mathrm{A}$ and $\mathrm{D}$ around a given bond $\mathrm{BC}$ if bond crossing after the WWW move is to be avoided. It follows from Eq. (2) that the rate of transformation from the configuration $\{L, I, J, R\}$ to the new configuration $\{L+1, I-1, J-1, R+1\}$ is given by

$$
W_{\{L, I, J, R\}}=P_{\{L, I, J, R\}} p_{\text {in,fi }} \exp \left(-\frac{E_{a}}{k_{B} T}\right),
$$

where we have introduced an activation energy barrier $E_{a}$ for any WWW move. Finally, the rate equation for the concentration of $k$-member rings may be written as

$$
\frac{d n_{k}(t)}{d t}=\sum_{L=N_{\min }}^{N_{\max }-1} \sum_{I=N_{\min ^{+1}}}^{N_{\max }} \sum_{J=N_{\min }+1}^{N_{\max }} \sum_{R=N_{\min }}^{N_{\max }-1} C_{\{L, I, J, R\}}^{k} W_{\{L, I, J, R\}},
$$

where the coefficients $C_{\{L, I, J, R\}}^{k}$ are defined by

$$
\begin{aligned}
C_{\{L, I, J, R\}}^{k}= & \delta_{L+1, k}+\delta_{I-1, k}+\delta_{J-1, k}+\delta_{R+1, k}-\delta_{L, k}-\delta_{I, k}-\delta_{J, k} \\
& -\delta_{R, k},
\end{aligned}
$$

with $\delta_{i, j}$ as the Kronecker symbol. The coefficient $C_{\{L, I, J, R\}}^{k}$ is therefore an integer between -4 and 4 . The actual value of this coefficient is equal to the number of rings with size $k$ generated with the rate $W_{\{L, I, J, R\}}$ minus the number of disappearing rings of size $k$ during a transition from $\{L, I, J, R\}$ to $\{L+1, I-1, J-1, R+1\}$. For example, $C_{\{4,4,4,4\}}^{4}$ is equal to -4 in the transition shown in Fig. 1 as all 4-member rings disappear in that configuration. The other two nonzero coefficients in that configuration are $C_{\{4,4,4,4\}}^{3}$ and $C_{\{4,4,4,4\}}^{5}$. They both are equal to 2 , as two three- and two 5-member rings were generated by the particular reaction shown in Fig. 1.

\section{SPATIALLY INHOMOGENEOUS CASE}

In order to simulate the process of amorphous spot generation or collapse in the crystalline matrix, we need to generalize our rate equation model to account for the inhomogeneous variation in temperature and ring concentrations along the phase-change material. In addition, we need to introduce explicitly the interface energy between the amorphous and crystalline phases. We will assume that the thickness of the phase-change material deposited on a substrate is sufficiently small for temperature and ring distributions to be constant through the depth of the film, depending only on the in-plane coordinates $x$ and $y$.

We coarse grain our atomistic model by dividing the $2 \mathrm{D}$ $\{x y\}$ space into cells, which are chosen to take the same 


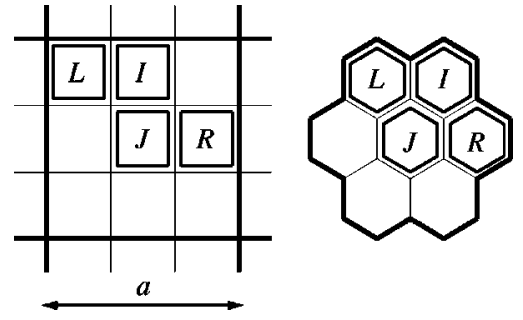

FIG. 2. A schematic illustration of the cell in our coarse grained model. Left-hand panel: the lattice constant $a$ is equal to three times that of the atomic lattice to incorporate four rings of the WWW bond switching event from Fig. 1. Right-hand panel: the configuration involved in WWW bond switching in a honeycomb lattice.

symmetry as the underlying crystalline lattice. For the fourfold coordinated system shown in Fig. 1, the cells would be arranged in a square lattice with the lattice constant equal to three times that of the atomic lattice to incorporate four rings, as shown in Fig. 2. This figure also illustrates the cell in our simulations of the three-fold coordinated honeycomb lattice, which will be discussed in the next section. Each cell is then regarded as homogeneous with uniform temperature $T_{m}$ and ring concentration $n_{k}^{m}$, where $m$ is the cell number. The rate equation for the $k$-member ring concentration $n_{k}^{m}$ is

$$
\frac{d n_{k}^{m}}{d t}=\frac{1}{2} \sum_{m^{\prime}, m^{\prime \prime}}\left(S_{m^{\prime}, m^{\prime \prime}} \sum_{L, I, J, R} c_{k ;\{L, I, J, R\}}^{m^{\prime}, m^{\prime \prime}} W_{\{L, I, J, R\}}^{m^{\prime}, m^{\prime \prime}}\right),
$$

where the coefficients $c_{k ;\{L, I, J, R\}}^{m^{\prime}, m^{\prime \prime}}$ are defined by

$$
\begin{aligned}
c_{k ;\{L, I, J, R\}}^{m^{\prime}, m^{\prime \prime}}= & \delta_{m, m^{\prime}}\left(\delta_{L+1, k}+\delta_{I-1, k}-\delta_{L, k}-\delta_{I, k}\right) \\
& +\delta_{m, m^{\prime \prime}}\left(\delta_{R+1, k}+\delta_{J-1, k}-\delta_{R, k}-\delta_{J, k}\right),
\end{aligned}
$$

and the rates $W_{\{L, I, J, R\}}^{m^{\prime}, m^{\prime \prime}}$ are given by

$$
W_{\{L, I, J, R\}}^{m^{\prime}, m^{\prime \prime}}=\frac{1}{Z} \frac{2 L I J R}{(I-1)(J-1)} n_{L}^{m^{\prime}} n_{I}^{m^{\prime}} n_{J}^{m^{\prime \prime}} n_{R}^{m^{\prime \prime}} p_{i n, f i} \exp \left(-\frac{E_{a}}{k_{B} T}\right) .
$$

The indices $m^{\prime}$ and $m^{\prime \prime}$ run over the $m$ th cell and its nearest neighbors. Since we require bond conservation within each cell, the WWW move in the inhomogeneous system involves either all four rings from the $m$ th cell $\left(m^{\prime}=m^{\prime \prime}=m\right)$, or two rings from the $m$ th cell and the other two rings from one of the neighboring cells $\left(m^{\prime}=m\right.$ and $m^{\prime \prime} \neq m, \quad m^{\prime \prime}=m$ and $m^{\prime} \neq m$ ), as expressed by Eq. (9). In the latter case the two rings in each cell are chosen such that one ring size increases while the other ring size decreases after the WWW move. The appropriate statistical weight for the intracell and intercell reactions is taken into account by the term $S_{m^{\prime}, m^{\prime \prime}}$ in Eq. (8), which is equal to 1 for the $\left(m^{\prime}=m^{\prime \prime}=m\right)$ case and to 0.25 otherwise.

The rate equation model accounts for all possible microscopic WWW moves that result in creation and destruction of a $k$-member ring in cell $m$, as seen on the right-hand side of Eqs. (8)-(10). For a given set of $n_{k}^{m}$ there could be many different microscopic ring configurations for many different WWW moves. To account for all possible configurations in
Eqs. (8)-(10) we use proper statistical factors and continuous ring concentrations to model the amorphous and crystalline phases. For a given microscopic configuration the underlying atomic aligment is known. Therefore, to be consistent, the correct statistical weight also includes the energy difference of the initial and final states, which is described by the term $p_{\text {in,fi }}$ in Eq. (10).

The amorphous-crystalline interface is described by introducing an environment-dependent energy penalty. For a $k$-member ring in the $m$ th cell this can be defined by

$$
E_{k}^{m}=E_{k}\left[1+\sum_{m^{\prime} \neq m} \lambda \Theta\left(n_{4}^{m^{\prime}}-n_{4}^{c}\right)\right],
$$

where $n_{4}^{m}$ is the concentration of four-member rings in the $m$ th cell, and $n_{4}^{c}$ is the concentration of four-member rings, above which the phase is considered as crystalline. $\Theta\left(n_{4}^{m}-n_{4}^{c}\right)$ is a step function equal to unity if the expression in brackets is positive, and equal to zero otherwise. The index $m^{\prime}$ runs over the nearest neighbors of the $m$ th cell. The energy penalty given by Eq. (11) suggests that the generation of non-four-member rings requires more energy in the crystalline phase than it does in the amorphous phase.

The total energy of the system can be decomposed in terms of bulk and interface contributions using Eqs. (1) and (11), and is given by

$$
\begin{aligned}
E_{\text {tot }} & =\sum_{k, m} E_{k} n_{k}^{m}+\lambda\left(\sum_{m \in \text { interface }}\left\{\sum_{k, m^{\prime} \neq m}\left[E_{k} n_{k}^{m} \Theta\left(n_{4}^{m^{\prime}}-n_{4}^{c}\right)\right]\right\}\right) \\
& =E_{\text {bulk }}+E_{\text {interface }} .
\end{aligned}
$$

The interface energy is proportional to parameter $\lambda$ and as we will see in the next section it influences recrystallization from the interfaces. By definition the interface energy depends on the number of crystalline cells in the nearest neighborhood to the amorphous cell. Therefore, it depends on the crystallographic orientation of the interface, i.e., on the crystal facet.

We illustrate the method by choosing the same energy penalties $E_{k}$ as those for amorphous silicon. They were obtained by analyzing the ring distributions in five different amorphous silicon structures that had been simulated by a Monte Carlo technique. ${ }^{23}$ We found that $95 \%$ of the energy of amorphous silicon structures could be accounted for by considering in Eq. (1) only 5-, 6-, 7-, and 8-member rings, with energy penalties $E_{5}^{\mathrm{Si}}=0.6 \mathrm{eV}, \quad E_{6}^{\mathrm{Si}}=0.0 \mathrm{eV}$, $E_{7}^{\mathrm{Si}}=-0.2 \mathrm{eV}, E_{8}^{\mathrm{Si}}=0.8 \mathrm{eV}$. The fact that $E_{7}^{\mathrm{Si}}$ is negative does not have any physical implications, as it is not possible to generate a state consisting solely of seven-member rings. Due to conservation of rings, the seven-member rings energy is balanced by those of five- and eight-member rings. The energy penalties in three-dimensional silicon are measured with respect to the six-member rings of the crystalline diamond lattice. Since the crystal structure in our model is the square lattice, the ring energies should be counted with respect to the four-member rings. Therefore, as a convenient first guess we have assigned the energy penalties as $E_{3}$ $=0.6 \mathrm{eV}, E_{4}=0.0 \mathrm{eV}, E_{5}=-0.2 \mathrm{eV}, E_{6}=0.8 \mathrm{eV}$.

The crystallization of amorphous structures usually occurs by either shrinking the amorphous phase due to the 


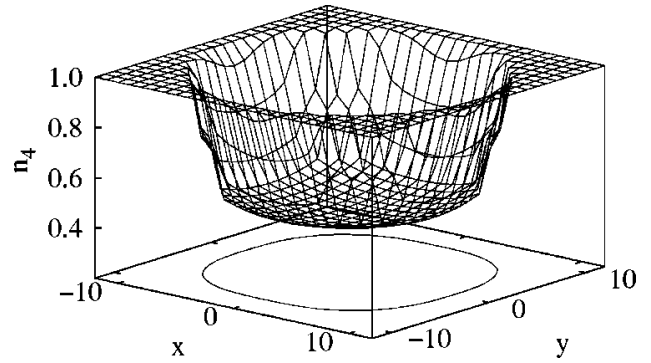

FIG. 3. Snapshot of an amorphous spot: concentration of fourmember rings in the system at $t_{0}$ when the pulse switched off. The contour line at the bottom is plotted for $n_{4}=0.75$.

amorphous-crystalline interface motion, or nucleation and growth of crystallites inside the amorphous phase. In our model only the first mechanism is considered. We chose $\lambda$ equal to 0.5 to ensure re-crystallization from the interfaces and the activation energy to be equal to $E_{a}=2 \mathrm{eV}$ in order to ensure a reasonable lifetime for the amorphous spot. This latter value is within the range of published experimental activation energies for the $\mathrm{Ge}-\mathrm{Sb}-\mathrm{Te}$ phase-change material. ${ }^{15}$ The parameter $n_{4}^{c}$ (Eq. (11)) was chosen to be equal to 0.75 . We also note here that the choice of $a \times a$ cell of course limits the size of the rings that can be considered. In Fig. 2 for the square lattice the coarse graining is shown for $a=3$. In principle, an eight-membered ring could be easily fitted within this cell, as its diameter would be equal to $2 a$. Nevertheless, this eight-member ring would have an enormous energy penalty in our two-dimensional square lattice, and this is the reason why we do not take into account ring sizes larger than the 6-member rings in our square lattice simulations. Nevertheless, if required, the size of $a$ can be increased to any value. The only criterion is that the proper statistical weight for the intracell and intercell reactions $S_{m^{\prime}, m^{\prime \prime}}$ should be taken into account.

\section{RESULTS}

We are interested in the lifetime and stability of an amorphous spot generated by a heat pulse. A finite size amorphous spot (typically with a radius of $10-100 \mathrm{~nm}$ ) always recrystallizes due to the energy difference between the crystalline and amorphous phases. The time scale of this recrystallization can be associated as the lifetime of the amorphous spot. For simplicity, we assume that heat pulse generates a temperature profile of Gaussian form,

$$
T(x, y, t)=T_{e} \exp \left(-\frac{x^{2}+y^{2}}{\sigma^{2}}\right) \exp \left(-\frac{\left(t-t_{0}\right) \Theta\left(t-t_{0}\right)}{\tau}\right)+T_{\text {sub }} .
$$

The prefactor $T_{e}$ is the excess temperature generated by the heat pulse, $T_{\text {sub }}$ is the substrate temperature, the coordinates $x$ and $y$ are measured from the pulse center in units of the cell size, $\sigma$ is the width of the pulse in cell size units, $t_{0}$ is the duration of the heat pulse, and $\tau$ is the decay time determined by the heat transport from the 2D layer to the substrate. We neglect any heat transport within the $\{x y\}$ plane.

During the time period $t<t_{0}$ the heat pulse amorphizes the material within a distance $\sigma$ from the center of the pulse. After switching off the heat pulse the material will not recrystallize during the decrease of the temperature to $T_{\text {sub }}$ if $\tau$ is small. The amorphous spot is characterized by a lower concentration of four-member rings than the surrounding crystalline material, as seen in Fig. 3. We note here that during the time period $t<t_{0}$ the system may be in the liquid phase. However, it is not the objective of the rate equation model to describe the liquid phase after melting. In the liquid phase the coordination number might be different to that of crystalline and amorphous phases and therefore we may not describe it as a network. For example, in case of silicon the coordination number in liquid is equal to six, whereas in the crystalline and amorphous phase it is four. Therefore, the rate equation model describes the crystalline and amorphous ("frozen liquid") states via Eqs. (8) and (13).

Snapshots of a simulated amorphization-crystallization process are shown in Figs. 4(a)-4(e). During the early stages of the amorphization process $\left(t<t_{0}\right)$ the spot has a circular shape corresponding to the shape of the temperature profile (Figs. 4(a)). In the latter stages the spot acquires a square

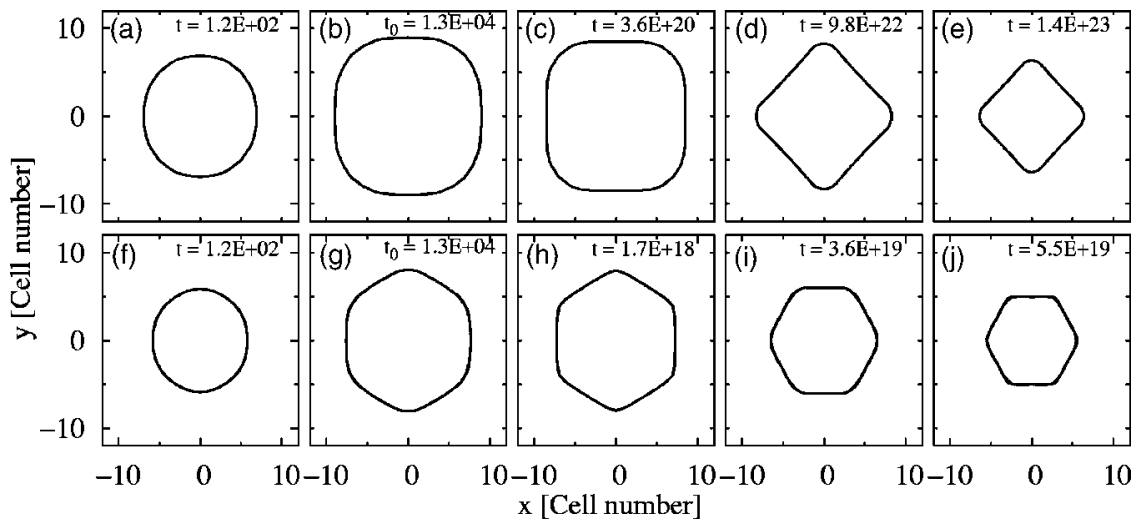

FIG. 4. Snapshots of "amorphization-recrystallization" computer experiments showing contour lines of 75\% of 4- and 6-member ring concentrations for square ((a)-(e)) and honeycomb ((f)-(j)) lattices, respectively. The amorphous spots at $t=t_{0}$ have a faceted shape according to the square lattice (b) and honeycomb lattice (g). Recrystallization process is mediated by the fastest moving facets ((d), (e)) and ((i), (j)). The substrate and excess temperatures have been chosen as $k_{B} T_{\text {sub }}=0.025 \mathrm{eV}$ and $k_{B} T_{e}=0.750 \mathrm{eV}$, respectively, with $t_{0}=1.3 \mathrm{E}$ +04 and $\tau=13$. 
shape in accordance with Eqs. (8) and (11), which show that the crystal facets with the lowest number of nearest neighbors for each amorphous cell (low energy facets) move faster. After the heat pulse is switched off at $t_{0}$, the amorphous spot is stable for a long time at room temperature $\left(k_{B} T=25 \mathrm{meV}\right)$, showing low energy facets (Fig. 4(c)). The changes in the shape of the amorphous spot are observed when the high energy crystal facets eventually move forward, shrinking the size of the spot. The high energy facets are oriented at $45 \mathrm{deg}$ with respect to the low-energy facets (Figs. 4(d) and 4(e)). Such a transformation of the spot shape is accounted for by Eqs. (8) and (11), which predict the fastest moving facets during crystallization to be those with the most number of nearest neighboring crystalline cells for each amorphous cell.

Faceting depends on the symmetry of the crystalline phase. To reveal such dependence we have also performed simulations in a three-fold coordinated system with a honeycomb structure, where the crystalline phase is characterized by six-member rings. According to the change of the crystal ring size in Eq. (11), the subscript 4 should be replaced by subscript 6 . The 2D space is now divided into cells, which have the same symmetry as the underlying honeycomb lattice, as shown in Fig. 2. The coefficients $S_{m^{\prime}, m^{\prime \prime}}$ describing the statistical weight for the intracell and intercell reactions in Eq. (8) are equal to 1 for the $m^{\prime}=m^{\prime \prime}=m$ case and to 0.166 otherwise. We assigned energy penalties $E_{k}$ the same as those for silicon, which were derived earlier in the previous section.

The stable amorphous spot now resembles the underlying honeycomb lattice, as illustrated in Fig. 4(g). During recrystallization the amorphous spot facets are rotated by $30 \mathrm{deg}$ compared to the stable amorphous spot (Figs. 4(i) and 4(j)). Therefore, we observed again that the recrystallization process is mediated by the fastest moving facets (Fig. 4). According to the definition of the facet (interface) energy given by Eq. (12), the fastest moving facets are those with the highest interface energy. Crystal faceting during crystallization is often observed in atomistic simulations ${ }^{14}$ and in real systems. ${ }^{30,31}$

The time step was chosen as ten times smaller than $\exp \left[E_{a} /\left(k_{B} T_{e}+k_{B} T_{\text {sub }}\right)\right]$ for the time period $t<t_{0}$. This ensures that by the given parameter set in our simulations the largest rate occurring on the right-hand side of Eq. (8) is smaller than $5 \times 10^{-2}$. After the switch of the pulse $\left(t>t_{0}\right)$ we used a dynamic time step. Namely, the time step was chosen in a way that the largest rate has never exceeded the value of $10^{-4}$ in Eq. (8). In the experiments the amorphous spots in phase-change materials are written during a time period of the order of nanoseconds $\left(t_{0}\right)$. A calibration of the time scale in the rate equation model to this value would mean the spot lifetime of the order of $10^{10} \mathrm{~s}(\sim 100$ years $)$ in the square lattice simulations, which is within the required stability period of 10 years for industrial phase-change data storage.

We have studied the dependence of the lifetime of the amorphous spot upon its size by controlling the width of the heat pulse through parameter $\sigma$ in Eq. (13). For constant values of the activation energy $E_{a}$ and the decay time $\tau$ we

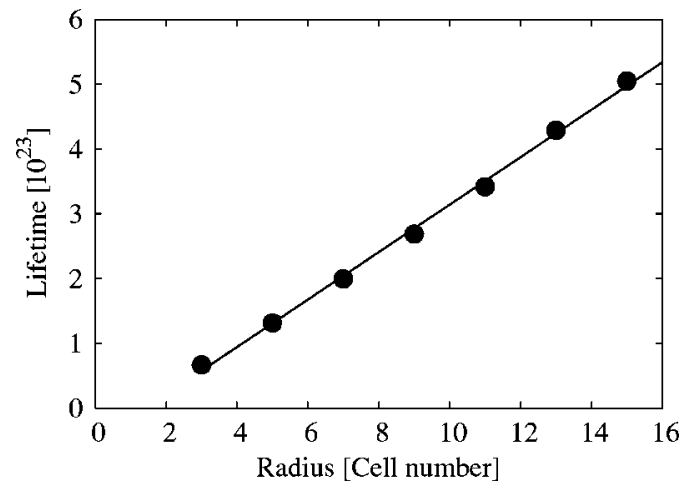

FIG. 5. Lifetime of amorphous spots in a 2D square lattice with activation energy $E_{a}$ equal to $2 \mathrm{eV}$.

have found that the lifetime is a linear function of the spot radius, if the latter is large compared to the cell size, as seen in Fig. 5. The linear dependence of the lifetime upon the spot radius is consistent with faceting during the crystallization process: the facets move nearly independently of each other, spreading as plane waves with constant speed. In practice, the linear dependence would most likely be violated due to facet pinning by defects and impurities. The linear dependence breaks down for spot sizes comparable to the cell size due to the coarse-grained nature of our model. The time scale of the amorphization-crystallization process in the square and honeycomb lattice simulations is somewhat different for the same parameter set. This is due to the difference in the number of nearest-neighbor cells appearing in Eq. (11).

\section{CONCLUSION}

We have developed a rate equation model, which describes the amorphization of a network material due to local heating, and its recrystallization after switching off the heat pulse. The model combines atomistic and continuum approaches by using microscopic parameters for the ring distributions and ring energetics, but continuous ring concentration variables are used to describe the amorphous and crystalline phases. Using this rate equation model we have studied the generation and collapse of amorphous spots within the crystalline matrix, processes that are central to phase-change data storage. We have found that the shape of the amorphous spot changes during its lifetime. It is determined by the low-energy crystal facets during the growth and long time period after, until it starts collapsing due to the motion of the high-energy facets. The motion of the highenergy facets leads to a linear dependence of the spot lifetime upon the spot size. The rate equation model could be generalized for the case of 3D network materials, assuming that the number of rings is conserved on average. However, further theoretical developments would be required to extend this model to non-network materials.

\section{ACKNOWLEDGMENTS}

The research is funded by Hewlett-Packard Laboratories (Palo Alto, California). Valuable discussions with Jim Brug, 
C. C. Yang, Gary Gibson, Chris Nauka, and Alison Chaiken are gratefully acknowledged. K. K. and V. M. B. are thankful to Adrian Sutton and Sebastian von Alfthan for inspiring discussions. The calculations were performed at the Oxford Supercomputing Centre and the Materials Modelling Laboratory (University of Oxford).
*Electronic address: krisztian.kohary@ materials.ox.ac.uk

${ }^{1}$ J. W. Toigo, Sci. Am., 282, 50 (2000).

${ }^{2}$ G. A. Gibson, A. Chaiken, K. Nauka et al., Appl. Phys. Lett. 86, 051902 (2005).

${ }^{3}$ A. Chaiken, G. Gibson, J. Chen, B. Yeh, J. Jasinski, Z. LilientalWeber, K. Nauka, C. Yang, D. Linding, and S. Subramanian (unpublished).

${ }^{4}$ A. Chaiken, G. A. Gibson, K. Nauka, C. C. Yang, B. S. Yeh, R. Bicknell-Tassuis, J. Chen, J. Jasinski, Z. Liliental-Weber, and D. D. Lindig, Paper HH.1.9, 2003 MRS Fall Meeting, Boston, MA, 2003 (unpublished).

${ }^{5}$ G. A. Gibson et al., Paper No. HH.2.6, 2003 MRS Fall Meeting, Boston, MA, 2003 (unpublished).

${ }^{6}$ T. Ohta and S. R. Ovshinsky, "Phase-Change Optical Data Storage Media," p. 310-326, in Photo-Induced Metastability in Amorphous Semiconductors, Edited. by A. V. Kolobov (WileyVCH, New York, 2003).

${ }^{7}$ S. von Alfthan, A. P. Sutton, A. Kuronen, and K. Kaski (unpublished).

${ }^{8}$ K. Gaertner and B. Weber, Nucl. Instrum. Methods Phys. Res. B 202, 255 (2003).

${ }^{9}$ L. A. Marques, L. Pelaz, M. Aboy, L. Enriquez, and J. Barbolla, Phys. Rev. Lett. 91, 135504 (2003).

${ }^{10}$ S. M. Nakhmanson and N. Mousseau, J. Phys.: Condens. Matter 14, 6627 (2002)

${ }^{11}$ N. Bernstein, M. J. Aziz, and E. Kaxiras, Phys. Rev. B 58, 4579 (1998).

${ }^{12}$ M. J. Caturla, T. Diaz de la Rubia, L. A. Marques, and G. H. Gilmer, Phys. Rev. B 54, 16683 (1996).

${ }^{13}$ C. R. S. da Silva and A. Fazzio, Phys. Rev. B 64, 075301 (2001).

${ }^{14}$ Y. Tu, J. Tersoff, G. Grinstein, and D. Vanderbilt, Phys. Rev. Lett.
81, 4899 (1998).

${ }^{15}$ S. Senkander and C. D. Wright, J. Appl. Phys. 95, 504 (2004).

${ }^{16}$ J. Wei and F. Gan, Thin Solid Films 441, 292 (2003).

${ }^{17}$ E. R. Meinders and C. Peng, J. Appl. Phys. 93, 3207 (2003).

${ }^{18}$ P. K. Khulbe, E. M. Wright, and M. Mansuripur, J. Appl. Phys. 88, 3926 (2000)

${ }^{19}$ E. M. Wright, P. K. Khulbe, and M. Mansuripur, Appl. Opt. 39, 6695 (2000).

${ }^{20}$ C. A. Volkert and M. Wuttig, J. Appl. Phys. 86, 1808 (1999).

${ }^{21}$ C. Peng, L. Cheng, and M. Mansuripur, J. Appl. Phys. 82, 4183 (1997).

${ }^{22}$ D. S. Franzblau, Phys. Rev. B 44, 4925 (1991).

${ }^{23}$ G. T. Barkema and N. Mousseau, Phys. Rev. B 62, 4985 (2000).

${ }^{24}$ N. A. Marks, N. C. Cooper, D. R. McKenzie, D. G. McCulloch, P. Bath, and S. P. Russo, Phys. Rev. B 65, 075411 (2002).

${ }^{25}$ F. Wooten, K. Winer, and D. Weaire, Phys. Rev. Lett. 54, 1392 (1985).

${ }^{26}$ K. A. Fichtorn and W. H. Weinberg, J. Chem. Phys. 95, 1090 (1991).

${ }^{27}$ D. R. Mason, R. E. Rudd, and A. P. Sutton, J. Phys.: Condens. Matter 16, S2679 (2004).

${ }^{28}$ D. H. Dunlap, P. E. Parris, and V. M. Kenkre, Phys. Rev. Lett. 77, 542 (1996)

${ }^{29}$ H. Cordes, S. D. Baranovskii, K. Kohary, P. Thomas, S. Yamasaki, F. Hensel, and J. H. Wendorff, Phys. Rev. B 63, 094201 (2001).

${ }^{30}$ N. A. Smith, H. H. Lamb, A. J. McGinnis, and R. F. Davis, J. Appl. Phys. 96, 4556 (2004).

${ }^{31}$ Z. G. Herro, B. M. Epelbaum, M. Bikcermann, P. Masri, and A. Winnacker, J. Cryst. Growth 262, 105 (2004). 